\title{
Low Temperature Debinding Process Using Oxygen Plasma for Flexible Printed Electronics
}

\author{
Young-In Lee* \\ Institute of Nano Sensor Technology, Hanyang University, Ansan 426-791, Republic of Korea
}

(Received July 18, 2012; Revised July 26, 2012; Accepted August 6, 2012)

\begin{abstract}
In this study, an oxygen plasma treatment was used as a low temperature debinding method to form a conductive copper feature on a flexible substrate using a direct printing process. To demonstrate this concept, conductive copper patterns were formed on polyimide films using a copper nanoparticle-based paste with polymeric binders and dispersing agents and a screen printing method. Thermal and oxygen plasma treatments were utilized to remove the polymeric vehicle before a sintering of copper nanoparticles. The effect of the debinding methods on the phase, microstructure and electrical conductivity of the screen-printed patterns was systematically investigated by FE-SEM, TGA, XRD and four-point probe analysis. The patterns formed using oxygen plasma debinding showed the well-developed microstructure and the superior electrical conductivity compared with those of using thermal debinding.
\end{abstract}

Keywords: Copper paste, Oxygen plasma treatment, Debinding, Polyimide, Flexible printed electronics

\section{Introduction}

Recently, fabrication of metallic circuit patterns on the surface of flexible substrate has become of increasing importance in flexible electronic device applications including display, solar cell array, radio frequency identification tags and chemical sensors [1-4]. Direct printing methods including screen, gravure, flexography, and inkjet printing have been intensively investigated as low-cost "greener" alternatives to traditional subtractive photolithography to create electrical connections [5-8]. In addition, these methods can write directly on various substrates (i.e., polyimide, polyethylene terephthalate, polycarbonate, and paper) to form flexible electronics [9-11].

The direct printed conductive features are generally created from metal pastes or inks consisted of metal particulates and polymers such as binders and dispersing agents. The polymers provide lubrication between particles, improve particle packing, assist in flow, and generally increase the handling strength of the printed pattern in the green state [12]. Following the direct printing of the pattern, the polymers should be completely removed from the patterns because they have a bad influence on the electrical conductivity of the patterns. Polymer burnout is the widely applied method for polymer removal and is commonly termed as thermal debinding. When polymeric films are utilized as a substrate of electronic application for the fabrication of flexible electronic devices, the thermal debinding could lead to a deformation of the substrate because their complete decomposition temperature is normally higher than the glass transition temperature of the polymeric films. Therefore, it is important to the development of a low temperature debinding method to realize various flexible electronics.

Oxygen plasmas provide an abundant source of energetic and chemically activated oxygen species that could remove carbon based organic materials because the oxidative degradation of polymers is well known $[13,14]$. Based on these characteristic, the oxygen plasma have been applied to clean and modify the surface of various polymeric substrates and to strip photoresists at low temperature [15-17]. Therefore, the oxygen plasma debinding might provide several advantages such as a low temperature debinding and a minimization of residual

*Corresponding Author : Young-In Lee, TEL: +82-31-400-4714, FAX: +82-31-418-6490, E-mail: youngin.fnmr@gmail.com 
organic materials compared to thermal debinding. However, the use of oxygen plasma treatment in fabrication of directly metallic circuit patterns on flexible substrates has not yet been reported.

In this study, we apply oxygen plasma treatment to remove the polymers in direct printed metallic circuit at a low temperature and demonstrate the effectiveness of oxygen plasma debinding by investigating and comparing the phase, microstructure and electrical conductivity of the copper patterns treated by the oxygen plasma and thermal debinding.

\section{Experimental Details}

In order to prepare copper pastes, the commercially available copper nanoparticles (average diameter: $80 \mathrm{~nm}$, NT BASE Co., Ltd., Korea) were mixed with polymeric vehicles which were prepared by mixing $0.15 \mathrm{~g}$ of ethyl cellulose (ethoxyl content: $48 \%$, Sigma-Aldrich), $1.5 \mathrm{~g}$ of $\alpha$-terpineol ( $>90 \%$, Sigma-Aldrich), $0.05 \mathrm{~g}$ of polyvinylpyrrolidone (Mw: 1,300,000, Sigma-Aldrich), and 0.5 $\mathrm{g}$ of diethylene glycol (>99.0\%, Daejung Chem. \& Metals Co., Ltd., Korea) using a paste mixer (UM-103, Japan Unix). The amount of the copper nanoparticle was fixed at $70 \mathrm{wt} \%$. Thick films from the copper paste were prepared by a screen-printing technique on a polyimide film (Kolon LV 200, $50 \mu \mathrm{m}$ thickness) using a screen mask (ST\#400, $10 \mu \mathrm{m})$. After screen-printing, the thick films were dried at $90^{\circ} \mathrm{C}$ for $60 \mathrm{~min}$ to remove a volatile organic solvent.

The plasma debinding of the screen-printed patterns was carried out using an inductively coupled plasma reactor (BEP-5066, Standard Asher RIE system, SNTEK) for $5 \mathrm{~min}$. The power and operating frequency were 300 $\mathrm{W}$ and $13.56 \mathrm{MHz}$, respectively. After pumpdown to 1.0 $\times 10^{-2}$ Torr, the oxygen with $150 \mathrm{sccm}$ of flow rate was fed into a chamber and the working pressure and temperature were controlled to $2.4 \times 10^{-2}$ Torr and $80^{\circ} \mathrm{C}$, respectively. Comparative samples were prepared by thermal debinding at $250^{\circ} \mathrm{C}$ for $60 \mathrm{~min}$ in air using a tube furnace. After the debinding step, the printed films were sintered at $250^{\circ} \mathrm{C}$ for $2 \mathrm{~h}$ in $\mathrm{H}_{2}$.

Field emission scanning electron microscope (FE-SEM, S4800, HITACHI Co.) and X-ray diffractometer (XRD, Rigaku, $\mathrm{CuK} \alpha$ ) were carried out to confirm the microstructures and phases of the printed films, respectively. The thermal behavior of the polymeric vehicle and the paste was investigated by thermogravimetric analysis (TGA, SDTQ-600, TA Instruments Co.). In addition, the resistivity was measured using sheet resistance obtained by a four-point probe (ATI CMT-SR2000N) and the thickness of the patterns.

\section{Results and Discussion}

The polymeric vehicles without copper nanoparticles were printed on a glass substrate and treated by heating and oxygen plasma to determine the debinding ability of the oxygen plasma treatment by observing residues after the debinding processes. As shown in Fig. 1(A), the oxygen plasma treatment completely removed the polymeric vehicle from glass substrate; on the other hand, when vehicle was thermally treated the residue on the glass substrate was clearly observed. Fig. 1(B) shows optical
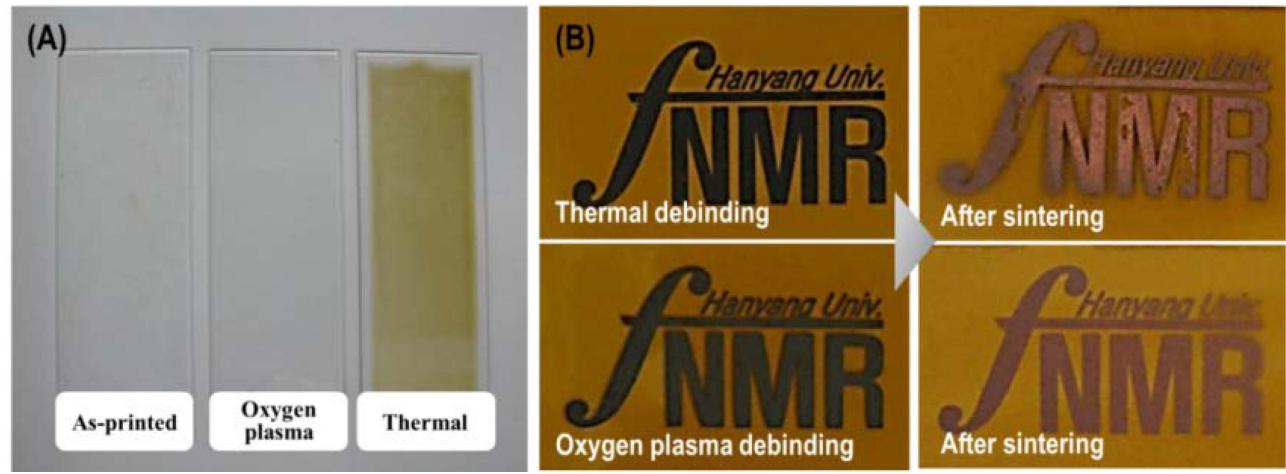

Fig. 1. Optical images of (A) polymeric vehicles printed on glass substrate and oxygen plasma (at $300 \mathrm{~W}$ for 5 min) or thermally $\left(\right.$ at $250^{\circ} \mathrm{C}$ for $1 \mathrm{~h}$ ) treated polymeric vehicles for decomposition of them and (B) screen-printed copper patterns on polyimide films after thermal or oxygen plasma debinding and further sintering steps. 

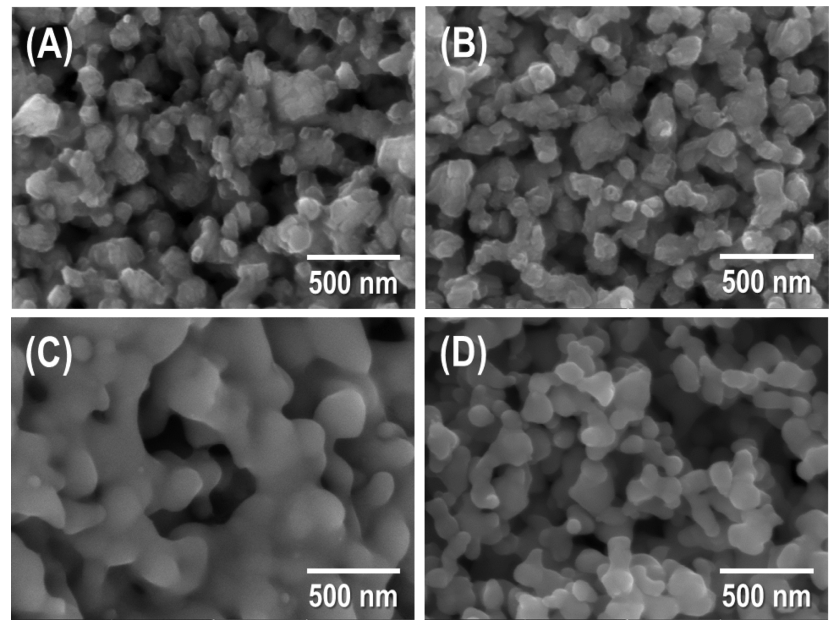

Fig. 2. FE-SEM images of (A) thermally and (B) oxygen plasma treated patterns for the decomposition of polymeric vehicles. (C) and (D) are FE-SEM images after the sintering of the (A) and the (B) at $250^{\circ} \mathrm{C}$ for $2 \mathrm{~h}$, respectively.

photograph of the screen-printed copper patterns on polyimide films before and after sintering. The patterns had clearly different colors and morphologies depending on the debinding methods. The pattern based on thermal debinding had a significantly irregular color and morphology compared to the pattern based on oxygen plasma debinding. This result directly indicates the effect of debinding method on the characteristics of the sintered conducting pattern.

FE-SEM analysis was performed to investigate the microstructure change in the screen-printed copper pattern each processing step. Fig. 2 shows the FE-SEM images of the screen-printed copper patterns after debinding the polymeric vehicles by heating and oxygen plasma and further sintering step. As shown in Fig. 2(A, B), after debinding the vehicles using different methods, there is no difference between them. However, the microstructure of the sintered samples was significantly different depending on the debinding methods. This difference could be explained by the residue of the polymeric vehicle remaining after the debinding step. For the pattern treated by oxygen plasma for the debinding, the relatively well-developed microstructure with the solid state neck formation and growth between the particles was observed after sintering step as shown in Fig. 2(D). On the other hands, for the pattern thermally debinding the polymeric vehicle, the pattern was covered with the residue of polymeric vehicle, and thus it is difficult to
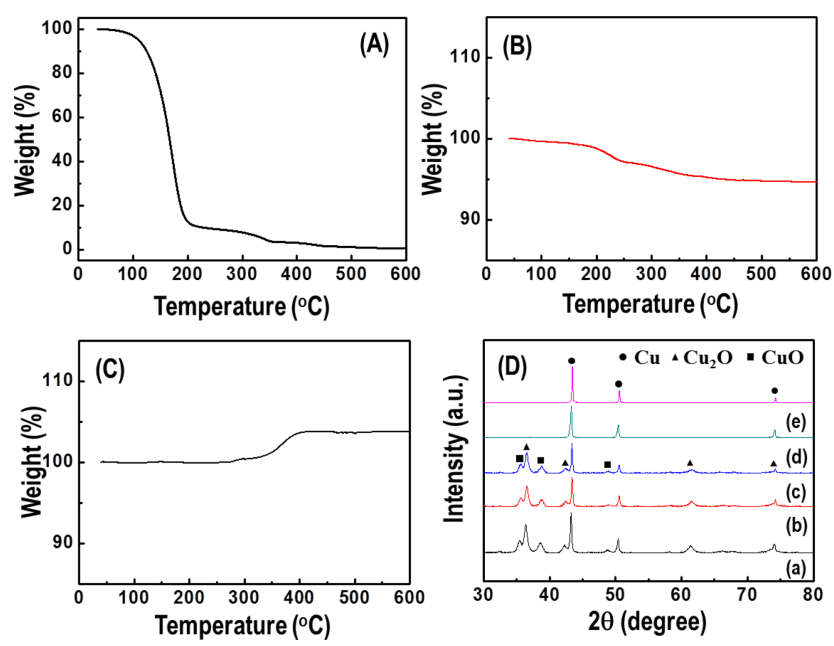

Fig. 3. Thermal gravimetric analysis graphs of (A) a polymeric vehicle and copper pastes treated by (B) thermal and (C) oxygen plasma processes for the decomposition of the polymeric vehicle. (D) XRD patterns of (a) commercial copper nanoparticles, and (b) thermally and (c) oxygen plasma treated patterns for debinding. (d) and (e) are XRD patterns after the sintering of the (b) and the (c), respectively.

observe the microstructure of the pattern [Fig. 2(C)].

Evidence for the difference of the pattern microstructure and the remained residue by the debinding methods was provided by TGA. Fig. 3(A) shows the TGA curve of the polymeric vehicle consisted of the binders and the dispersing agents. The sample was heated in an air atmosphere from room temperature to $600^{\circ} \mathrm{C}$ with a ramping rate of $10^{\circ} \mathrm{C} / \mathrm{min}$. As shown in Fig. 3(A), the weight of the sample rapidly decreased at temperature between 100 and $200^{\circ} \mathrm{C}$, but all materials were decomposed over $500^{\circ} \mathrm{C}$. However, when polymer films were used as the substrates for flexible electronics, decomposing the polymeric vehicles in the conductive paste at the temperature over $250^{\circ} \mathrm{C}$ is not suitable because the polymer films commonly have the lower glass transition temperature than $250^{\circ} \mathrm{C}$ [18]. Therefore, based on the TGA result, debinding the polymeric vehicle at that temperature to prevent the substrate deformation consequentially remains the residue of the polymeric vehicle. TGA results of the copper pastes treated by different type of debinding methods are presented in Fig. 3(B, C). The curve for the thermally debinding paste indicated that the weight loss started at a temperature of approximately $180^{\circ} \mathrm{C}$ and completed at about $500^{\circ} \mathrm{C}$ resulting from the decomposition of the residue as shown in Fig. 3(B). In the case of the copper paste treated by oxygen plasma, the weight of 
the sample remained constant until around $300^{\circ} \mathrm{C}$ and then increased by $5 \%$ between $300^{\circ} \mathrm{C}$ and $400^{\circ} \mathrm{C}$ due to the air oxidation of the copper nanoparticles [Fig. 3(C)]. The TGA results, which are obtained from the samples treated by heating and oxygen plasma for debinding the polymeric vehicle, clearly shows that oxygen plasma can completely remove the polymeric vehicle compared with heating.

The XRD peaks of the bare copper nanoparticles and the screen-printed copper patterns after debinding and further sintering are shown in Fig. 3(D). The bare copper nanoparticles have diffraction peaks of copper, cuprous oxide and cupric oxide. The presence of cuprous and cupric oxide peaks might be attributed to oxide formed by surface oxidation and during the preparation of the paste. As shown in Fig. 3[D (b, c)], the debinding steps were for nothing in the crystal structure of the patterns due to the low processing temperature. After sintering step at $250^{\circ} \mathrm{C}$ for $2 \mathrm{~h}$ in $\mathrm{H}_{2}$, all samples had face-centered cubic copper structures with diffraction peaks from the (111), (200) and (220) planes (JCDPS \#: 04-0836), and no peaks for any other phases were detected, indicating the high purity of the product. Fig. 4 shows the resistivity of the screen-printed copper pattern formed using the different debinding methods after the sintering step at $250^{\circ} \mathrm{C}$ for $2 \mathrm{~h}$ in $\mathrm{H}_{2}$. The resistivities of thermally and oxygen plasma treated copper patterns were 124 and 16.6 $\mu \Omega \cdot \mathrm{cm}$, respectively (bulk copper: $1.67 \mu \Omega \cdot \mathrm{cm}$ ). Such relatively low specific resistance of the sample prepared using oxygen plasma debinding method compared with thermal debinding was highly related to the complete removal of the organic vehicle and the relatively well-

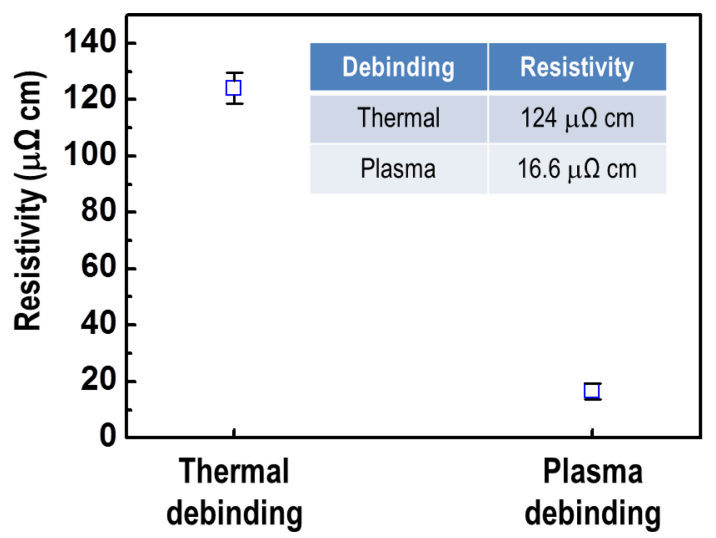

Fig. 4. Specific resistances of screen-printed conductive copper patterns according to debinding processes. sintered microstructure. Based on the above results, we know that the debinding methods on microstructure and electrical conductivity of the direct printed copper patterns have a significant impact and the efficiency of oxygen plasma for the removal of polymeric vehicles is clearly demonstrated.

\section{Conclusion}

In conclusion, we have developed a facile and versatile technique to remove polymeric vehicles in direct printed conductive copper patterns on a flexible substrate at a low temperature by introducing an oxygen plasma treatment as a debinding method. While the residue of the polymeric vehicle remained in the screenprinted pattern after thermal debinding step at $250^{\circ} \mathrm{C}$, the polymeric vehicle was completely removed by the oxygen plasma debinding process. The patterns formed using oxygen plasma debinding has the well-developed microstructure and the superior electrical conductivity compared with those of using thermal debinding due to the great debinding ability of oxygen plasma. This debinding technique using the oxygen plasma could be used for various types of inks or pastes as well as diverse direct printing methods including screen, gravure, and offset printing for flexible printed electronics.

\section{Acknowledgment}

This work was financially supported by the Pioneer Research Center Program through the National Research Foundation of Korea (2011-0001645) funded by the Ministry of Education, Science and Technology (MEST) and was supported by Grant No. 10031768 from the Ministry of Knowledge Economy (MKE), Republic of Korea.

\section{References}

[1] Y. Chen, J. Au, P. Kazlas, A. Ritenour, H. Gates and M. McCreary: Nature, 423 (2003) 136.

[2] F. C..Krebs, M. Jфrgensen, K. Norrman, O. Hagemann, J. Alstrup, T. D. Nielsen, J. Fyenbo, K. Larsen and J. Kristensen: Sol. Energy Mater. Sol. Cells, 93 (2009) 422.

[3] D.-Y. Shin, Y. Lee and C. H. Kim: Thin Solid Films, 517 (2009) 6112.

[4] S. Y. Hwang, H. Y. Jung, J.-H. Jeong and H. Lee: Thin 
Solid Films, 517 (2009) 4104.

[5] W. Su, P. Li, L. Yao, F. Yang, L. Liang and J. Chen: Chem Phys Chem, 12 (2011) 1143.

[6] J. Puetz and M. A. Aegerter: Thin Solid Films, 516 (2008) 4495.

[7] Y.-I. Lee and Y.-H. Choa: J. Mater. Chem., 22 (2012) 12517.

[8] Y.-S. Cho, S.-J. Son, Y. K. Kim, K. O. Ohung and C. J. Choi: J. Kor. Powd. Met. Inst., 17 (2010) 390 (Korean).

[9] B. K. Park, D. Kim, S. Jeong, J. Moon and J. S. Kim: Thin Solid Films, 515 (2007) 7706.

[10] T. H. J. van Osch, J. Perelaer, A. W. M. de Laat and U. S. Schubert: Adv. Mater., 20 (2008) 20, 343.

[11] Y.-L. Tai and Z.-G. Yang: J. Mater. Chem., 21 (2011) 5938.
[12] R. K. Enneti, S. J. Park, R. M. German and S. V. Atre: Mater. Manuf. Processes, 27 (2012) 103.

[13] M. Myers: J. Phys. B, 2 (1969) 393.

[14] J. L. Bolland: Proc. R. Soc. London Ser. A, 186 (1946) 218.

[15] M. A. Hartney, D. W. Hess and D. S. Soane: J. Vac. Sci. Technol. B, 7 (1989) 1.

[16] Y. Kim, M. R. Baklanov, T. Conard, M. de Potter and S. Vanhaelemeersch: J. Electrochem. Soc., 146 (1999) 1549.

[17] Y.-S Goo, Y.-I. Lee, N. Kim, K-J. Lee, B. Yoo, S.-J. Hong, J.-D. Kim and Y.-H. Choa: Surf. Coat. Technol., 205 (2010) S369.

[18] C. E. Wilkes, J. W. Summers and C. A. Daniels: PVC Handbook, Hanser Gardner Publications, Inc., USA (2005). 